\title{
Promoção da saúde e prevenção da gravidez na adolescência: relato de experiência
}

\author{
Promoting the health of adolescents: an experience report \\ Promoción de la salud de los adolescentes: un informe de experiencia
}

Vanessa Bernardo da Silva Souza1*, Fernanda Barbara Valadão1, Ilda Estefani Ribeiro Marta1, Sonia Regina Jurado ${ }^{1}$, Mara Cristina Ribeiro Furlan¹.

\section{RESUMO}

Objetivo: Relatar a experiência de um projeto de um programa de educação tutorial de enfermagem (PETEnfermagem) com intuito de realizar promoção da saúde aos adolescentes e prevenção da gravidez na adolescência da região leste de Mato Grosso do Sul. Relato da experiência: Trata-se do relato de um projeto direcionado a 59 adolescentes com 13 anos de idade ou mais. Seis encontros foram realizados uma vez por semana em uma escola pública, durante os meses de agosto e setembro de 2019. O projeto utilizou diversas técnicas lúdicas e dinâmicas para instruir e levar as informações sobre puberdade, higiene corporal, prevenção de Infecções Sexualmente Transmissíveis (ISTs), projeções de futuro, gravidez na adolescência, métodos contraceptivos, entre outros. Considerações finais: Por meio da execução do projeto foi possível disseminar informações sobre promoção à saúde dos adolescentes e instruir, profissionalmente, maneiras de prevenir uma gravidez na adolescência. Além disso, houve troca de conhecimentos e experiências de vidas entre os alunos, pais e estudantes de enfermagem.

Palavras-chave: Gravidez na adolescência, Promoção da saúde, Educação em saúde, Enfermagem.

\begin{abstract}
Objective: To report the experience of a project of a tutorial education program (PET-Nursing) with the aim of promoting health among adolescents and preventing teenage pregnancy in the eastern region of Mato Grosso do Sul. Experience report: This is the report of a project aimed at 59 adolescents aged 13 years or older. Six meetings were held once a week in a public school, during the months of August and September 2019. The project used several playful and dynamic techniques to instruct and convey information about puberty, body hygiene, prevention of Sexually Transmitted Infections (STIs), future projections, teenage pregnancy, contraceptive methods, among others. Final considerations: Through the execution of the project, it was possible to disseminate information on promoting the health of adolescents and professionally instruct ways to prevent teenage pregnancy. In addition, there was an exchange of knowledge and life experiences between students, parents and nursing students.
\end{abstract}

Key words: Pregnancy in adolescence, Health promotion, Health education, Nursing.

\section{RESUMEN}

Objetivo: Informar la experiencia de un proyecto de un programa de educación tutorial (PET-Enfermería) con el objetivo de promover la salud en adolescentes y prevenir el embarazo adolescente en la región oriental de

1 Universidade Federal de Mato Grosso do Sul (UFMS), Três Lagoas - MS.

*E-mail: va.bernardosilva@hotmail.com

SUBMETIDO EM: 6/2021

ACEITO EM: 7/2021

PUBLICADO EM: 8/2021 
Mato Grosso do Sul. Informe de experiencia: Este es el informe de un proyecto dirigido a 59 adolescentes de 13 años o más. Se realizaron seis reuniones una vez a la semana en una escuela pública, durante los meses de agosto y septiembre de 2019. El proyecto utilizó varias técnicas lúdicas y dinámicas para instruir y transmitir información sobre la pubertad, la higiene corporal, la prevención de Infecciones de Transmisión Sexual (ITS).), proyecciones de futuro, embarazo adolescente, métodos anticonceptivos, entre otros. Consideraciones finales: Mediante de la ejecución del proyecto, se logró difundir información sobre la promoción de la salud de los adolescentes e instruir profesionalmente las formas de prevenir el embarazo adolescente. Además, hubo un intercambio de conocimientos y experiencias de vida entre estudiantes, padres y estudiantes de enfermería.

Palabras clave: Embarazo en adolescencia, Promoción de la salud, Educación en salud, Enfermería.

\section{INTRODUÇÃO}

A adolescência é definida como o momento de transição entre a infância e a fase adulta. Um período marcado por transformações físicas, psicológicas e sociais (SCHAEFER R, et al., 2018). Nesse ciclo da vida, as relações sexuais aumentam consideravelmente o risco de Infecções Sexualmente Transmissíveis (ISTs). Em 2019, o Brasil concentrou mais de 40\% das novas infecções por ISTs, em adolescentes e adultos jovens, de 15 a 19 anos, caracterizando um aumento de 53\%, em relação ao período de 2004 a 2013 (COSTA MIF, et al., 2019).

Além do risco para aquisição de ISTs, o sexo desprotegido pode levar a gravidez, que é considerada fator de risco nessa faixa etária, para mudanças sociais e de saúde, tais como depressão, evasão escolar, uso de drogas, problemas na relação familiar, situações de violência e isolamento. Para o bebê de mãe adolescente há riscos como: prematuridade, baixo peso ao nascer, atraso no desenvolvimento (REGO MH, et al., 2018).

De acordo com dados do Ministério da Saúde (2019), a taxa mundial de gravidez na adolescência foi estimada em 44 nascimentos para cada mil adolescentes entre 15 e 19 anos no período de 2016. Para as Américas, esse indicativo foi de 48,6/1000 nascidos. No Brasil, essa taxa foi de 56,4/1000 nascidos.

Para redução de ISTs e gravidez na adolescência, se faz necessário fortalecer o vínculo da escola com a comunidade. Temas como a anatomia e fisiologia do corpo humano, métodos de prevenção da gravidez precoce e ISTs são essenciais para a promoção da saúde da população. Após o ambiente familiar, a escola é responsável por complementar a educação, onde é possível acompanhar a fase de desenvolvimento dos adolescentes e contribuir para que as orientações pertinentes sejam realizadas por meio de discussões relacionadas aos temas estabelecidos (ALMEIDA RAAS, et al., 2017).

A escola é um ambiente que fortalece o processo educativo para integração do adolescente ao meio social, gerando relações que se estabelecem por toda a vida. Esse espaço se caracteriza como facilitador para projetos que orientem assuntos diversos como educação em saúde, violência e efetivação de políticas públicas (SOUZA VP, et al., 2020).

De acordo com Barbosa LU (2019), a educação sexual no espaço escolar vem se apresentando como uma intervenção necessária, uma vez que contribui para a construção da personalidade dos indivíduos e oportuniza questionamentos, reflexões e discussões que resgatam a marca humana da sexualidade: amor, afeto, favorecendo a qualidade nas relações sexuais e sociais. Para Zompero AF (2018), a educação para sexualidade é de extrema importância para formação do estudante, tanto no aspecto pessoal como social, e a escola deve contribuir para esta formação.

A importância de espaços confortáveis voltados aos adolescentes, para que eles possam compartilhar seus questionamentos e adquirir informações sobre a sexualidade e orientações relacionadas aos métodos contraceptivos são primordiais para a prevenção das ISTs e gravidez indesejada (SILVA TT, et al., 2015).

Dessa forma, este estudo possui objetivo de relatar a experiência de um projeto com intuito de realizar promoção à saúde aos adolescentes e prevenção da gravidez na adolescência em ambiente escolar. 


\section{RELATO DE EXPERIÊNCIA}

Este relato de experiência foi elaborado a partir de um projeto de extensão realizado pelo programa de educação tutorial de enfermagem (PET Enfermagem). A ação foi desenvolvida no segundo semestre do ano de 2019, com seis integrantes do grupo e dois acadêmicos voluntários do curso de enfermagem.

O projeto foi realizado junto a 59 adolescentes com 13 anos e mais, que frequentavam um projeto social para reforço escolar em um município do Estado de Mato Grosso do Sul. Inicialmente foi realizado um encontro com os 59 pais/responsáveis no qual a equipe responsável pelo projeto apresentou os objetivos e as ações propostas. Após esclarecimentos de dúvidas, a totalidade dos participantes se manifestaram favoráveis ao desenvolvimento do projeto.

As atividades com os adolescentes ocorreram no período matutino e vespertino, uma vez por semana durante os meses de agosto e setembro, sendo realizados cinco encontros, com duração aproximada de três horas.

Os temas aplicados no projeto dizem respeito a metas de vida, anatomia e fisiologia do sistema reprodutor feminino e masculino, higiene pessoal, prevenção de gravidez na adolescência, IST's e o Sistema Único de Saúde (SUS).

No primeiro encontro com os adolescentes, realizou-se a apresentação da equipe e informou-se o cronograma de cada encontro. Além disso, desenvolveu-se a dinâmica "caixa-correio", que possuía o objetivo de sanar dúvidas de forma anônima e aplicar pré-testes sobre questões relacionadas aos próximos encontros do projeto, com intuito de mensurar o nível de conhecimento a respeito das ações a serem desenvolvidas.

Ainda, realizou-se uma dinâmica de conhecimento dos temas propostos no decorrer dos encontros, chamada de "brainstorm" (tempestade de ideias). Os adolescentes foram divididos em dois grupos e cada um escreveu em uma cartolina o que sabiam sobre os temas, em seguida, apresentaram as informações dando início a discussão. No início do encontro os adolescentes estavam inibidos. Porém neste momento se fez perceptível o interesse e interrogações acerca do conteúdo a ser explorado.

No segundo encontro, trabalhou-se o tema: metas e projeto de vida e foi desenvolvida uma atividade chamada "Cine PET". Assistiu-se o filme "A Procura da Felicidade", que aborda perseverança nos objetivos e metas de vida. Ao término do filme, realizou-se uma dinâmica em que cada adolescente recebeu um papel com um traço ao meio que representaria a linha do tempo de sua vida. Nessa linha do tempo, descreveram os principais acontecimentos de sua vida até o momento atual. A partir de então, os adolescentes foram indagados sobre quais as metas para alcançar os objetivos que concretizarão seu projeto de vida, proporcionando interação e discussão sobre sonhos e possíveis barreiras no decorrer do caminho, despertando motivação e perspectivas entre eles. Houve boa participação dos adolescentes, e ficou evidenciado que muitos acontecimentos positivos e negativos tem a capacidade de influenciá-los nos planos futuros.

Conhecendo o corpo e higiene, foi o tema do terceiro encontro. A proposta se desenvolveu de forma expositiva e dialogada. Iniciou-se com a exposição das imagens ilustrativas indicando cada estrutura, função e fisiologia básica do sistema reprodutor feminino e masculino e foi enfatizada a importância de fazer a higiene corporal. Durante essa atividade os adolescentes apresentavam-se concentrados a cada detalhe exposto, demonstravam-se mais à vontade para esclarecer dúvidas, houve aumento dos questionamentos de forma natural, o que fortaleceu o vínculo e confiança.

No quarto encontro, o tema trabalhado foi gravidez na adolescência. A primeira dinâmica proposta para este encontro possuía o intuito dos adolescentes simularem o cuidado com um "bebê". Um adolescente do sexo feminino e um do sexo masculino foi convidado para participar, entregou-se uma boneca para que no decorrer da dinâmica ao som do choro, fossem realizados alguns cuidados que incluíam: preparar à alimentação, trocar a fralda, dar banho, enquanto o "bebê" chorava. Ao final da aula questionou-se sobre a percepção dos alunos sobre o conteúdo ofertado e as dificuldades de cuidar de uma criança durante a adolescência. 
Realizou-se ainda uma atividade com auxílio do kit sobre métodos contraceptivos e álbum seriado sobre as ISTs do Ministério da Saúde. Os materiais continham exemplos de métodos contraceptivos, dentre eles, dispositivo intrauterino (DIU), diafragma, cartela de contraceptivos orais, cartela de pílula do dia seguinte, contraceptivos injetáveis, anel vaginal, preservativos masculinos e preservativos femininos.

Foi apresentado cada método contraceptivo disponibilizado pelo SUS, seu modo de ação, uso e a importância da indicação por enfermeiro ou médico. Em seguida, realizou-se a prática de como deve ser utilizada a camisinha masculina e feminina com modelos anatômicos dos órgãos genitais masculinos e femininos, confeccionados em silicone. A cada método contraceptivo apresentado as indagações surgiram, tais como, o uso concomitante dos preservativos feminino e masculino, o motivo do preservativo masculino possuir sabor, o que constata a relevância da ação para essa faixa etária.

Os temas do quinto encontro foram: IST's e o SUS. Foi apresentado aos alunos as características das principais ISTs, suas formas de manifestação, como identificá-las, formas de transmissão e prevenção. Realizou-se uma dinâmica intitulada "Pega ou não Pega? " com o objetivo de reconhecer a IST e sua forma de contaminação, a cada resposta, os adolescentes foram questionados sobre a veracidade da afirmação.

Ainda nesse encontro, realizou-se uma discussão sobre como o SUS se articula no apoio à saúde do adolescente e seus direitos. Também se aplicou um pós-teste, elaborado com as mesmas questões aplicadas no pré-teste, para avaliar o aumento de conhecimento sobre os temas trabalhados. Houve ainda um momento para que os adolescentes relatassem suas percepções em relação ao projeto.

No encerramento os adolescentes expressaram gratidão pelo desenvolvimento das atividades, relataram efeitos positivos da aprendizagem proporcionada pelo projeto em suas vidas e escreveram mensagens de estímulo para a continuidade do mesmo.

\section{DISCUSSÃO}

A ação de extensão permitiu trabalhar temas juntos aos adolescentes para prevenção da gravidez na adolescência por meio de técnicas lúdicas e dinâmicas. Esse tipo de ação educativa é pertinente, pois favorece o acadêmico, futuro profissional de saúde, compreender e vivenciar as particularidades inerentes dessa população em todo o contexto que ela está inserida, fomentando através da comunicação, uma abordagem direcionada para a promoção e prevenção, concernente à área da saúde (SANTOS JS, et al., 2020).

O ambiente escolar tem se tornado um local privilegiado para a promoção de ações de educação em saúde, possibilitando autonomia do autocuidado, através de uma abordagem ampliada e específica para a população adolescente, onde eles possam estar desinibidos para apresentar suas dúvidas e expressar suas opiniões, estabelecendo assim um canal de comunicação aberto para o diálogo (FREITAS BHBM, et al., 2019).

Visando a inserção na fase adulta, se faz importante definir o papel social, profissional e familiar ainda durante a adolescência. As experiências pessoais, sociais e culturais vividas ao longo do tempo podem alterar o desenvolvimento do projeto, permanecendo em adaptação até sua concretização (SILVEIRA KSS, et al., 2015).

A escola oferece oportunidades para diversas intervenções, dado que pode contribuir para o engajamento dos adolescentes em vários momentos inclusive no recreio, sendo utilizado como estratégia para facilitar a atividade física em muitas instituições de ensino (COSTA BGG, et al., 2019).

Afim de considerar a informação como um fator de proteção e promoção a saúde, é necessário que as orientações sejam disseminadas de maneira adequada e integral, esclarecendo efeitos negativos como as consequências de sexo desprotegido e uso de drogas, porém citando que existem pontos prazerosos, que podem evoluir para amarguras e fatalidades no futuro. Deste modo, acredita-se que os adolescentes poderão agir de forma mais consciente frente às pressões externas (VIERO VSF, et al., 2015). 
Diante da quantidade de informações de caráter empírico que surgem de fontes variadas, somente a família não pode sanar, muitas vezes todas as dúvidas e orientações sobre sexualidade e ISTs. Os Parâmetros Curriculares Nacionais (PCN) elaborados pelo Ministério da Educação (MEC), inclui esses temas no programa, dentro dos Temas Transversais (SILVA R, 2015).

A comunidade adolescente frequenta pouco os serviços de saúde, e, em vista disso, a escola é um espaço que favorece ações de promoção e proteção da saúde, mediante a carência de informações. É importante ressaltar que os enfermeiros exercem papel protagonista no planejamento e na organização de atividades que colaboram para o fortalecimento da relação entre a saúde e a escola, com o objetivo de minimizar os efeitos nocivos a essa população (BRITO MFSF, et al., 2019).

Embora à abordagem da sexualidade humana, esteja em desenvolvimento, essa temática conduz consigo mitos, preconceitos e contrastes que atrapalham as reflexões sobre esse assunto junto aos adolescentes. Sendo assim, essa questão, que ainda é um tabu, precisa ser melhor pesquisada tanto pelos profissionais de saúde como os da educação, para que os adolescentes tenham condições de exercer sua cidadania e assumir responsabilidades para a preservação dos seus direitos legais (HIGA EFR, et al., 2015).

A gravidez em mães adolescentes, possui maior probabilidade de complicações como, síndromes hipertensivas, trabalho de parto prematuro, doença trofoblástica fetal (CORTEZ MB, et al., 2021). Ainda, parte dessa população realiza abortos inseguros, fato que contribui para a mortalidade materna e problemas de saúde que podem se tornar permanentes (DARROCH JE, et al., 2016).

Para o Ministério da Saúde (2017), a maneira de reduzir o quantitativo de gravidez não desejada é o investimento por parte do Ministério da Saúde em políticas de promoção à saúde visando como um dos objetivos o planejamento reprodutivo. Distribuir a Caderneta de adolescentes (CSA) do sexo feminino e masculino é umas das iniciativas, além disso, estimular o adolescente a ser o protagonista do seu desenvolvimento. Além disso, procurar estimular o adolescente a ser o personagem principal do seu desenvolvimento e distribuir pílula combinada, anticoncepção de emergência, minipílula, anticoncepcional injetável mensal e trimestral, diafragma, assim como preservativo feminino e masculino. E recentemente o DIU de Cobre, incluindo as adolescentes dentro desse público. O DIU é um método que dura 10 anos, e não necessita que a adolescente tenha que lembrar de tomar pílula diariamente.

Sobre as ISTs, vale destacar que elas estão entre as causas mais comuns de doenças no mundo, que seguem gerando várias complicações e até mesmo sequelas como infertilidade, perda fetal, gravidez ectópica e morte prematura, bem como infecções em recém-nascidos e lactentes (PINHEIRO PLL e CADETE MMM, 2019).

Por fim, as atividades lúdicas representaram o principal elo de fortalecimento do vínculo estabelecido entre os componentes do grupo e os adolescentes. Cada adolescente dispôs da oportunidade de expressar seu ponto de vista e discutir sobre os temas em destaque. Ações realizadas em nível escolar devem ser estimuladas, pois demonstram possuir potencial para obtenção de resultados importantes para mudanças no estilo de vida e aquisição de conhecimentos por adolescentes.

\section{REFERÊNCIAS}

1. ALMEIDA RAAS, et al. Conhecimento de adolescentes relacionados às doenças sexualmente transmissíveis e gravidez. Revista Brasileira de Enfermagem, 2017. 70(5): 1033-1039.

2. BARBOSA LU, et al. Percepción de adolescentes sobre sexualidad y salud reproductiva: la escuela como espacio para la educación sexual. Cultura de los Cuidados (Edición digital), 2019; 23 (55).

3. MINISTÉRIO DA SAÚDE. Saúde e mais três ministérios firmam carta compromisso para prevenção da gravidez na adolescência. Brasília. Ministério da Saúde. Brasil, 2019. Disponível em: https://antigo.saude.gov.br/noticias/agenciasaude/45196-saude-e-mais-tres-ministerios-firmam-carta-compromisso-para-prevencao-da-gravidez-naadolescencia. Acesso em: 15 de julho de 2019.

4. MINISTÉRIO DA SAÚDE. Departamento de Vigilância, Prevenção e Controle das IST, do HIV/Aids e das Hepatites Virais. Álbum Seriado das Infecções Sexualmente Transmissíveis (IST). Brasil, 2017. Disponível em: http://www.aids.gov.br/pt-br/pub/2017/album-seriado-das-infeccoes-sexualmente-transmissiveis-ist. Acesso em: 15 de julho de 2019. 
5. BRITO MFSF, et al. Fatores associados ao estilo de vida de estudantes do ensino médio de escolas públicas. Revista Gaúcha de Enfermagem, 2019. 40: 1-8.

6. CORTEZ MB, et al. Análise das complicações clínico-obstétricas em gestantes adolescentes segundo a Classificação de Robson. Revista Enfermagem UERJ, 2021. 29, p. e49539.

7. COSTA BGG, et al. Efeito de uma intervenção sobre atividade física moderada a vigorosa e comportamento sedentário no tempo escolar de adolescentes. Revista Brasileira de Epidemiologia, 2019. 22: 1-12.

8. COSTA MIF, et al. Determinantes sociais da saúde e vulnerabilidades às infecções sexualmente transmissíveis em adolescentes. Revista Brasileira de Enfermagem, 2019. 72(6): 1595-1601.

9. DARROCH JE, et al. Costs and benefits of meeting the contraceptive needs of adolescents: Guttmacher Institute; 2016.

10. FREITAS BHBM, et al. Oficina educativa com adolescentes sobre hanseníase: relato de experiência. Revista Brasileira de Enfermagem, 2019; 72(5): 1421-1425.

11. HIGA EFR, et al. A intersetorialidade como estratégia para promoção da saúde sexual e reprodutiva dos adolescentes. Interface, 2015. 19(1): 879-891.

12. PINHEIRO PLL, Cadete MMM. O conhecimento dos adolescentes escolarizados sobre o papiloma vírus humano: revisão integrativa. Enferm. Glob, 2019. 18(56): 603-663.

13. REGO MH, et al. Resiliência e gravidez na adolescência: uma revisão integrativa. Psic. Saúde \& Doenças, 2018; 19(3): 710-123.

14. RESTREPO AM, et al. Análisis de los elementos de mercadeo social implícitos en campañas de prevención de embarazo en adolescentes. Rev. Fac. Nac. Salud Pública, 2018; 36(2): 18-27.

15. SANTOS JS, et al. Processo de comunicação em saúde da enfermagem com o adolescente: abordagem do Event History Calendar. Revista Brasileira de Enfermagem, 2020; 73(3).

16. SCHAEFER R, et al. Políticas de Saúde de adolescentes e jovens no contexto luso-brasileiro: especificidades e aproximações. Ciênc. Saúde Coletiva, 2018; 23(9): 2849-2858.

17. SILVA R. Quando a escola opera na conscientização dos jovens adolescentes no combate às DSTs. Educar em Revista, 2015. 57: 221-238.

18. SILVA TT, et al. Contracepção em adolescentes nos últimos 15 anos: perspectiva de um Centro de Atendimento a Jovens. Nascer e Crescer, 2015; 24(3): 108-111.

19. SILVEIRA KSS, et al. Projetos futuros de adolescentes privados de liberdade: implicações para o processo socioeducativo. Psicol. teor. Prat, 2015; 17(2): 52-63.

20. SOUZA VP, et al. Protagonismo de adolescentes no planejamento de ações para prevenir a violência sexual. Texto contexto - Enfermagem, 2020. 29.

21. VIERO VSF, et al. Educação em saúde com adolescentes: análise da aquisição de conhecimentos sobre temas de saúde. Escola Anna Nery, 2015. 19(3): 484-490.

22. ZOMPERO AF, et al. A temática sexualidade nas propostas Curriculares no Brasil. Revista Ciências e Ideias, 2018; $9(1), 101-114$. 